\title{
Overcoming limitations of self-report: an assessment of fear of weight gain in anorexia nervosa and healthy controls using implicit association tests
}

Tiana Borgers ${ }^{1 \dagger}$, Nathalie Krüger ${ }^{2 \dagger}$, Silja Vocks ${ }^{2}$, Jennifer J. Thomas ${ }^{3}$, Franziska Plessow ${ }^{4}$ and Andrea S. Hartmann ${ }^{2^{*}}$ (D)

\begin{abstract}
Background: Fear of weight gain is a characteristic feature of anorexia nervosa (AN), and reducing this fear is often a main target of treatment. However, research shows that $20 \%$ of individuals with AN do not report fear of weight gain. Studies are needed that evaluate the centrality of fear of weight gain for AN with a method less susceptible to deception than self-report.

Methods: We approximated implicit fear of weight gain by measuring implicit drive for thinness using implicit association tests (IATs). We asked 64 participants (35 AN, 29 healthy controls [HCs]) to categorize statements as prodieting vs. non-dieting and true vs. false in a questionnaire-based IAT, and pictures of underweight vs. normal-weight models and positive vs. negative words in a picture-based IAT using two response keys. We tested for associations between implicit drive for thinness and explicitly reported psychopathology within AN as well as group differences between $\mathrm{AN}$ and $\mathrm{HC}$ groups.

Results: Correlation analyses within the AN group showed that higher implicit drive for thinness was associated with more pronounced eating disorder-specific psychopathology. Furthermore, the AN group showed a stronger implicit drive for thinness than HCs in both IATs.

Conclusion: The results highlight the relevance of considering fear of weight gain as a continuous construct. Our implicit assessment captures various degrees of fear of weight gain in AN, which might allow for more individually tailored interventions in the future.
\end{abstract}

Keywords: Anorexia nervosa, Implicit association test, IAT, Drive for thinness, Fat phobia, Implicit association, Fear of weight gain, Feeding and eating disorders

\footnotetext{
* Correspondence: andrea.hartmann@uni-osnabrueck.de

†Tiana Borgers and Nathalie Krüger are Joint first authors.

${ }^{2}$ Unit of Clinical Psychology and Psychotherapy, Institute of Psychology,

Osnabrück University, Knollstr. 15, 49069 Osnabrück, Germany

Full list of author information is available at the end of the article
}

(c) The Author(s). 2021 Open Access This article is licensed under a Creative Commons Attribution 4.0 International License, which permits use, sharing, adaptation, distribution and reproduction in any medium or format, as long as you give appropriate credit to the original author(s) and the source, provide a link to the Creative Commons licence, and indicate if changes were made. The images or other third party material in this article are included in the article's Creative Commons licence, unless indicated otherwise in a credit line to the material. If material is not included in the article's Creative Commons licence and your intended use is not permitted by statutory regulation or exceeds the permitted use, you will need to obtain permission directly from the copyright holder. To view a copy of this licence, visit http://creativecommons.org/licenses/by/4.0/. The Creative Commons Public Domain Dedication waiver (http://creativecommons.org/publicdomain/zero/1.0/) applies to the data made available in this article, unless otherwise stated in a credit line to the data. 


\section{Plain English summary}

While fear of weight gain is the main known reason for food intake restriction in individuals with anorexia nervosa, some individuals deny experiencing it. It is relevant to know whether fear of weight gain a) actually does not exist in these individuals, b) cannot be consciously perceived, or c) is denied, as this might impact choice of intervention strategies and reduce treatment drop-out found in this group. As self-reports might be biased, other assessment methods less susceptible to deception need to be implemented. Since the reasons for food intake restriction vary across and within individuals with anorexia nervosa, fear of weight gain should be viewed as a dimensional variable in this context. Therefore, the aim of our study was to examine associations between implicit fear of weight gain and other eating disorder symptoms. We assessed fear of weight gain by measuring drive for thinness, with the latter not being an identical but closely related construct that has shown to differentiate between individuals with anorexia nervosa reporting vs. denying fear of weight gain in explicit measures. Therefore, we used two implicit tasks in a sample of individuals with anorexia nervosa and healthy controls. Results indicate that implicit drive for thinness is associated with a range of eating disorder-specific and general psychopathological symptoms. In addition, individuals with anorexia nervosa showed greater implicit drive for thinness than healthy controls. These results illustrate the importance of assessing implicit drive for thinness in individuals with anorexia nervosa for an ultimate adaptation of therapeutic interventions according to the individual level of fear of weight gain, both explicitly reported and implicitly measured.

\section{Background}

Anorexia nervosa (AN) is a severe and chronic mental disorder with one of the highest mortality rates among all mental illnesses [1]. The efficacy of psychological interventions is limited [2,3] and needs to be increased through an updated understanding of the central and potentially maintaining features of this disorder [4]. Body image disturbance-one of the key symptoms-is characterized by a multifactorial pattern of inappropriate attitudes and emotions toward the body, such as body dissatisfaction or fear of weight gain [5]. While body dissatisfaction in AN has been intensively studied [6], research on fear of weight gain (fat phobia, FP) is still lacking. Cognitive-behavioral theories have acknowledged that FP is particularly relevant for body selfschema activation, leading to disorder-specific cognitive biases and behaviors, with resulting distortions leading to a reinforcement of this fear [7]. Hence, early research stated that FP is central to the phenomenology of $\mathrm{AN}$, and it was assumed to be the main reason for food intake restriction and a central diagnostic criterion in the Diagnostic and Statistical Manual of Mental Disorders fourth edition (DSM-IV-TR) [8]. In the DSM-5, however, an explicit expression of FP is no longer a sine qua non criterion of $\mathrm{AN}$, as long as persistent behavior that counteracts weight gain is demonstrated [9]. Indeed, research suggests that 15-20\% of individuals with AN do not report FP $[10,11]$. These individuals with nonfat-phobic AN (NFP-AN) have shown lower scores on the restraint, eating concern, weight concern, and shape concern subscales of the Eating Disorder Examination (EDE [12]) and on the body dissatisfaction subscale of the Eating Disorder Inventory - 2 (EDI-2 [13]) than individuals with fat-phobic AN (FP-AN [10, 14, 15]). Additionally, they have exhibited fewer bulimic symptoms [14]. With regard to general psychopathology, individuals with NFP-AN typically present with lower depression and trait anxiety compared to individuals with FP-AN $[11,15,16]$. Furthermore, individuals with NFP-AN show significantly higher drop-out rates [11], higher standardized mortality rates [17], and reduced insight into their condition [11]. Accordingly, differences between individuals with FP-AN and NFP-AN may also be due to a lack of conscious awareness of the underlying FP [18]. Other reasons for differences in reported FP might be due to difficulties in identifying and describing their emotions (i.e., alexithymia [19]) or a tendency to deny both their low weight and AN [20] and potentially also FP in avoidance of treatment. Thus, a closer assessment of FP is essential in order to either modify treatment strategies depending on individual levels of FP or to resolve underlying shape and weight concerns in individuals denying or lacking awareness of FP, increasing efficacy of psychological interventions.

The aforementioned studies have mainly assessed FP by self-report $[21,22]$, which is problematic due to its susceptibility to intentional and unintentional deceptions as outlined above [18]. Implicit measurement methods might provide a solution for a more reliable assessment of FP [23]. One of the most common instruments is the Implicit Association Test (IAT [24]). The original IAT asks individuals to categorize stimuli into four categories and category-combinations. The faster the categorization of stimuli to one of the four categories (two opposing ones on each side), the stronger the implicit association of the two constructs on one side [25]. In clinical research, disorder-specific implicit attitudes have been successfully assessed, for instance, in social anxiety disorder [26], specific phobias [27], and obsessivecompulsive disorder [28].

In eating disorders (EDs), the IAT has been successfully employed to measure implicit ED-related attitudes [29-32]. To date, only one study has used the IAT to assess drive for thinness in individuals with FP-AN and 
NFP-AN, avoidant/restrictive food intake disorder (ARFI D), and healthy controls (HCs [33]). Izquierdo et al. [33] employed a questionnaire-based IAT (qIAT [34]) to assess the association between the categories pro-dieting/ non-dieting and true/false statements, and a picturebased IAT (pIAT) to assess the association between the categories underweight/normal-weight models and positive/negative words. Drive for thinness is not identical to FP but a closely related construct that has been shown to differentiate between individuals with AN reporting vs. denying FP in explicit measures of drive for thinness $[10,11,35]$. Therefore, Izquierdo et al. [33] examined implicit drive for thinness as an approximation for implicit FP using two IATs. The results revealed stronger associations between pro-dieting and true statements in participants with both FP-AN and NFP-AN. In contrast, a stronger association between pro-dieting and false statements was observed in HCs. In the pIAT, HCs demonstrated a stronger association between underweight models and negative words than did individuals with FP-AN and NFP-AN. While individuals with FPAN and NFP-AN differed in their self-reported FP, groups did not differ in their implicit association between pro-dieting and true statements in the qIAT. Additionally, both groups showed a similar difference from $\mathrm{HCs}$ in the pIAT, highlighting comparable implicit biases. However, the study did not take a dimensional approach, as it did not examine the association between implicit drive for thinness and psychopathology.

As illustrated above, studies investigating AN and FP are mainly based on a categorization into FP-AN vs. NFP-AN. However, taxometric studies [36, 37] have pointed mainly to a dimensional interpretation of ED pathology. Since ED pathology in general has shown to present dimensionally [36], and specific aspects, such as delusionality of appearance-related beliefs, vary widely across individuals with AN [38], dimensional models may contribute to a better understanding of the heterogeneity of $\mathrm{AN}$, resulting in stronger implications for considering FP as a continuous variable [36, 37]. This in turn would allow for a promotion of specific dimensional assessments and individually adapted treatments of individuals with AN on a continuum of FP. Therefore, the current study aimed to test whether implicitly measured drive for thinness, as a differentiating variable for FP, is associated with explicitly reported ED-specific and general psychopathology within AN, complementing the categorial findings of Izquierdo et al. [33] with a novel dimensional approach. As there is no comparable research approach on implicit drive for thinness in individuals with AN from a different culture than the United States (US) - American cultural background, the present study also aimed to extend these previous findings [33] to a German sample. Participants underwent a pIAT as well as a qIAT (see [33]), accordingly including pictures of underweight and normal-weight models as well as pro-dieting and non-dieting statements. A stronger bias toward underweight models or pro-dieting statements would represent implicit drive for thinness, while a stronger bias toward normal-weight models and nondieting statements would be interpreted as healthy. Additionally, the participants completed a set of self-report measures regarding ED-specific and general psychopathology. We hypothesized that (1) stronger implicit drive for thinness would be associated with higher scores on ED-specific and general psychopathology in the AN group and that (2) participants with AN, but not HCs, would exhibit an implicit drive for thinness in the IATs.

\section{Methods}

\section{Participants and recruitment}

Participants were recruited upon admission to outpatient and residential facilities, via advertisements in newspapers and on thematic websites, from the student population, and postings in therapeutic and counseling institutions. Inclusion criteria were female gender, age $\geq$ 15 years, sufficient knowledge of the German language, and absence of neurological diseases (relevant for other parts of the project). The inclusion criteria for the AN group were a score of $\geq 2$ on the SCOFF questionnaire [39], a screening instrument for identifying EDs, followed by a diagnosis of AN in the Structured Clinical Interview for DSM-IV (SCID; German version [40]). This DSM-IV SCID version [40] was applied, as the German version of the DSM-5 SCID [41] was first released in 2019. In order to comply with DSM-5 criteria of AN, we omitted the amenorrhea criterion and allowed a diagnosis of AN in the absence of FP if persistent behaviours counteracting weight gain were present in the setting of low weight and lack of recognition of associated medical consequences. Exclusion criteria for the AN group were a current psychotic and/ or manic episode, substance misuse or related addiction, and a body mass index (BMI) of 18.5 to $25 \mathrm{~kg} / \mathrm{m}^{2}$. For $\mathrm{HCs}$, exclusion criteria were any current or lifetime mental disorder and a BMI below $18.5 \mathrm{~kg} / \mathrm{m}^{2}$. After the screening of $N=118$ interested participants (HC: $n=62$; AN: $n=56$ ), $n=51$ were excluded due to not meeting inclusion criteria (in $\mathrm{HC}$ : age or BMI too low, or presence of mental disorder; in AN: age too low or BMI too high). After diagnostic assessment, another participant needed to be excluded for not meeting diagnostic criteria for AN. A total of $N=66$ participants took part in the study (HC: $n=29$; AN: $n=$ 37). Two participants with $\mathrm{AN}$ had to be excluded after participation in the study due to missing data, resulting in $N=64$ participants (HC: $n=29$; AN: $n=35$ ). A power analysis indicated that we had the power to detect 
medium to large effect sizes $(f=0.38)$ in the main analyses between participants with AN and HCs.

\section{Procedure}

First, a telephone prescreening was completed. Participants provided written informed consent (along with the parents if participants were minors) and underwent the following two diagnostic clinical interviews conducted by the senior author: The SCID for DSM-IV [40] and the EDE [12]. The IATs were conducted as part of a larger research project, which involved the following series of assessments: First, a picture was taken of each participant, which was used within the experimental tasks following the IAT paradigms, and a conjoint analysis was conducted. This paradigm has been described elsewhere [42]. The two IAT paradigms followed. Then eyetracking [43], encephalography, and electromyography paradigms were conducted (the two latter yielded still unpublished data). Finally, participants completed a questionnaire battery (see Questionnaires). At the end of the study, participants were informed about the objectives of the study and received a reimbursement of 70 Euros. The study procedure was approved by the University's ethics committee.

\section{Implicit association tests}

The two tasks were identical to those used by Izquierdo et al. [33], with the contents professionally translated into German. They were programmed and presented using Presentation 20.1 (Neurobehavioral Systems Inc., Berkeley, CA, USA) on a Dell Latitude E5520 laptop $(2.40 \mathrm{GHz}, 4.00 \mathrm{~GB})$ on a $16.5 \mathrm{in}$. HD WLED screen (resolution $1920 \times 1080$, color depth 32 bit). In both tasks, items were presented in random order in the middle of the screen, and participants were asked to categorize them into categories or category-combinations, indicated in the upper corners of the screen, via keystrokes. In two blocks, two sets of categories were displayed in the upper corners. The strength of association between two combined categories within an individual was operationalized as the speed with which the individual categorized items accordingly. The stronger the association, the easier and faster the categorization [32]. For both IATs, we followed the established block structure of Greenwald, Nosek, and Mahzarin [44]. To avoid sequence effects [45], two randomly assigned pIAT and qIAT versions were created, with different sequences of blocks $(59.4 \%$ of participants completed version 1 and $40.6 \%$ version 2 ).

\section{Picture-based implicit association test (pIAT)}

Categories presented in the pIAT included underweight models or normal-weight models and positive words or negative words [32,33]. Presented stimuli comprised five pictures of underweight vs. normal-weight models, and five words with a positive valence (e.g., "happy") vs. words with a negative valence (e.g. "awful"). For example, a faster categorization of a picture of an underweight model into the category underweight when combined with the category positive words (as compared to the opposite pairing) would be interpreted as implicit FP. Comparable with psychometrics of previous studies [44], the pIAT obtained an internal consistency of $r=.62$ $(p<.001)$.

\section{Questionnaire-based implicit association test (qIAT)}

The qIAT used in this study is conceptually based on the qIAT by Yovel and Friedman [34] and Izquierdo et al. [33]. The four categories pro-dieting vs. non-dieting statements and true vs. false statements each comprised five statements that could be categorized accordingly, e.g., "I am terrified of gaining weight" vs. "My weight rarely enters my mind" and "I'm participating in an experiment" vs. "I'm climbing a steep mountain". In this case, a faster categorization of a pro-dieting statement into the pro-dieting category, when combined with the category true statements, would be interpreted as implicit FP. In this study, the qIAT demonstrated an internal consistency of $r=.70(p<.001)$, which is in line with previous studies [34].

\section{Questionnaires}

\section{The SCOFF questionnaire (SCOFF [39])}

The SCOFF is a screening tool for EDs and was employed in the current sample to detect potential participants with psychopathological tendencies toward AN. It contains five questions addressing core features of AN and bulimia nervosa. In this study, we used German translations previously validated by Berger et al. [46] and Richter et al. [47]. The internal consistencies in German samples varied between $\alpha=.45$ and $\alpha=.66[46,47]$.

\section{Structured Clinical Interview for DSM-IV (SCID; German- language version [40])}

The SCID is a structured clinical interview used to diagnose DSM-IV Axis I disorders. In the current sample it was used for the diagnosis of AN and comorbid mental disorders. The interrater reliabilities were satisfactory to $\operatorname{good}\left(0.61 \leq r_{\text {icc }} \leq 0.83\right)$.

\section{Eating Disorder Inventory - (EDI-2; German-language version [13])}

The EDI-2 is a questionnaire assessing ED pathology. It consists of 11 subscales, of which the following were considered relevant as they are closest to fat-phobic psychopathology and used for further analyses in this study: body dissatisfaction, bulimia and drive for thinness. Items are rated on a six-point Likert scale from never 
(1) to always (6), and sum values were calculated as subscale scores. Internal consistencies of the EDI-2 subscales used in the present study were good to excellent $(.73 \leq \alpha \leq .94)$.

\section{Eating Disorder Examination (EDE; German-language version 17.0D [12])}

As a structured interview, the EDE assesses specific ED pathology on four subscales: restraint, eating concern, weight concern, and shape concern. All four subscales were considered relevant and used for further analyses in this study. Items are rated on a seven-point Likert scale from feature is not present (0) to feature is present every day/ to an extreme degree (6), and mean values were calculated as subscale scores. In the current sample, internal consistencies for the subscales were good to excellent $(.70 \leq \alpha \leq .95)$.

\section{Beck Depression Inventory-II (BDI-II; German-language version [48])}

This questionnaire assesses the level of depressive psychopathology. It consists of 21 items, each containing four statements varying in intensity or frequency, regarding the last two weeks. A total sum score was calculated for further analyses. In this study, the total score demonstrated an excellent internal consistency $(\alpha=.94)$.

\section{State-Trait Anxiety Inventory (STAl; German-language version [49])}

The STAI serves to capture fear as a state (state anxiety) and fear as a trait (trait anxiety). The trait version of the scale (Trait Anxiety scale, STAI-T) contains 20 items, which are based on statements regarding how the participants generally feel on four-point rating scales estimating frequencies from almost never (1) to almost always (4). A total sum score was calculated for the analyses. In this study, the internal consistency of the STAI-T was excellent $(\alpha=.96)$.

\section{Data analysis}

Data preparation and statistical analyses were conducted in Microsoft Excel (Microsoft, Redmond, USA) and SPSS 25 (IBM, Armonk, USA). To compare demographic characteristics, ED-specific psychopathology and general psychopathology between groups, we conducted two-sample $t$-tests and Mann-Whitney $U$-tests.

Data preparation of IAT data was based on the improved algorithm criteria by Greenwald et al. [44] and included adjustments depending on reaction times and incorrect responses, as well as calculation of the test values, the D-scores. Mean D-scores for each group in each IAT provided information about the direction as well as the strength of the association. The D-scores in this study are positive if the associations between pro- dieting statements and true statements or underweight models and positive words are stronger than the associations between pro-dieting statements and false statements or underweight models and negative words. The D-scores can be interpreted equivalently to Cohen's $d$ effect sizes (small: $d=0.2$; medium: $d=0.5$; large: $d=0.8$ [50]). In this study, the internal consistency of each IAT was calculated as the correlation between a D-score based on the practice blocks (Blocks 3 and 6) and a D-score based on the critical blocks (Blocks 4 and 7; see [44]).

To test our hypothesis that IAT D-scores would be positively correlated with ED-specific and general psychopathology in the AN group, we calculated Pearson correlation coefficients across all participants with AN to analyze interrelations of IAT D-scores with BDI-II and STAI-T scores. Relationships with non-parametric variables, such as BMI, EDE subscale scores (restraint, eating concern, weight concern, and shape concern), and EDI-2 subscale scores (body dissatisfaction, bulimia, and drive for thinness), were calculated using Spearman correlation coefficients. Furthermore, to test our hypothesis that participants with AN would exhibit significantly higher pIAT and qIAT D-scores than the HC group, we conducted two-sample $t$-tests.

\section{Results}

\section{Sample characteristics}

Fourteen participants with AN (40\%) had at least one comorbid disorder, with the major depressive disorder being the most common (17.14\%). As expected, participants with AN scored significantly higher on explicit measures of ED-specific and general psychopathology. As per study design, average BMI was significantly lower in the AN group than in HCs. Detailed sample characteristics are depicted in Table 1.

Associations of implicit drive for thinness with ED-specific and general psychopathology in participants with AN Within the AN group, medium positive correlations emerged between implicit drive for thinness (pIAT Dscores) and EDE eating concern and EDE shape concern. In addition, the analyses yielded significant medium to large positive correlations between implicit drive for thinness (qIAT D-scores) and ED-specific psychopathology measures, i.e., all four EDE subscales as well as EDI-2 drive for thinness and EDI-2 body dissatisfaction (Table 2). No significant correlations were found between D-scores of qIAT or pIAT and general psychopathological measures.

\section{Group differences in implicit drive for thinness} Picture-based implicit association test

There was a significant group difference in pIAT D-scores between participants with $\mathrm{AN}(M=-.13, S D=.60)$ and 
Table 1 Differences in demographic, anthropometric, and clinical variables between AN and HC

\begin{tabular}{|c|c|c|c|c|c|c|}
\hline & & $\begin{array}{l}\text { AN } \\
n=35\end{array}$ & $\begin{array}{l}\mathrm{HC} \\
n=29\end{array}$ & Test statistic & $p$-value & Effect size \\
\hline \multicolumn{7}{|c|}{ Demographic and anthropometric characteristics } \\
\hline Age (years) & $M(S D)$ & $24.80(9.23)$ & $23.28(2.52)$ & $U=428$ & .281 & $r=.14$ \\
\hline Relationship status & & & & $x^{2}=17.70^{* * *}$ & $<.001$ & $\varphi=.53$ \\
\hline In a relationship & $n(\%)$ & $7(20.0)$ & $21(72.4)$ & - & - & - \\
\hline Not in a relationship & $n(\%)$ & $28(80.0)$ & $8(27.6)$ & - & - & - \\
\hline University entry qualification & & & & $x^{2}=3.05$ & .081 & $\varphi=.22$ \\
\hline Obtained & $n(\%)$ & $29(82.9)$ & $28(96.6)$ & - & - & - \\
\hline Not obtained & $n(\%)$ & $6(17.1)$ & $1(3.4)$ & - & - & - \\
\hline Body mass index & $M(S D)$ & $15.87(2.85)$ & $21.41(2.19)^{\mathrm{a}}$ & $U=18^{* * *}$ & $<.001$ & $r=.82$ \\
\hline \multicolumn{7}{|c|}{ Eating disorder-specific psychopathology } \\
\hline Illness duration (years) & $M(S D)$ & $9.03(5,29)^{b}$ & - & - & - & - \\
\hline \multicolumn{7}{|l|}{ Comorbid disorders ${ }^{\mathrm{e}}$} \\
\hline $\mathrm{n}$ with $\geq 1$ comorbidities & $n(\%)$ & $14(40.0)$ & - & - & - & - \\
\hline MDD & $n(\%)$ & $6(17.14)$ & - & - & - & - \\
\hline Social anxiety disorder & $n(\%)$ & $4(11.43)$ & - & - & - & - \\
\hline Specific phobia & $n(\%)$ & $3(8.57)$ & - & - & - & - \\
\hline Panic disorder with agoraphobia & $n(\%)$ & $1(2.86)$ & - & - & - & - \\
\hline PTSD & $n(\%)$ & $1(2.86)$ & - & - & - & - \\
\hline OCD & $n(\%)$ & $3(8.57)$ & - & - & - & - \\
\hline BDD & $n(\%)$ & $1(2.86)$ & - & - & - & - \\
\hline \multicolumn{7}{|l|}{ EDE subscales } \\
\hline Restraint & $M(S D)$ & $3.17(1.74)$ & $.19(.53)^{d}$ & $U=46^{* * *}$ & $<.001$ & $r=.79$ \\
\hline Eating concern & $M(S D)$ & $2.49(1.44)$ & $.04(.12)^{d}$ & $U=19.5^{* * *}$ & $<.001$ & $r=.86$ \\
\hline Weight concern & $M(S D)$ & $2.45(1.47)$ & $.49(.60)^{d}$ & $U=87^{* * *}$ & $<.001$ & $r=.73$ \\
\hline Shape concern & $M(S D)$ & $3.14(1.64)^{c}$ & $.52(.65)^{d}$ & $U=65^{* * *}$ & $<.001$ & $r=.69$ \\
\hline \multicolumn{7}{|l|}{ EDI-2 subscales } \\
\hline Drive for thinness & $M(S D)$ & $31.09(8.56)$ & $13.34(6.13)$ & $U=80^{* * *}$ & $<.001$ & $r=.72$ \\
\hline Bulimia & $M(S D)$ & $14.8(6.86)$ & $10.62(3.69)$ & $U=322^{*}$ & .012 & $r=.31$ \\
\hline Body dissatisfaction & $M(S D)$ & $38.74(8,72)$ & $26.21(10.09)$ & $U=172.5^{* * *}$ & $<.001$ & $r=.57$ \\
\hline \multicolumn{7}{|l|}{ General Psychopathology } \\
\hline Total score & $M(S D)$ & $23.60(10.16)$ & $5.24(4.93)$ & $t=-9.44^{* * *}$ & $<.001$ & $d=2.23$ \\
\hline Trait-Anxiety & $M(S D)$ & $56.15(9.45)^{c}$ & $35.79(9.51)$ & $t=-8.49^{* * *}$ & $<.001$ & $d=2.18$ \\
\hline
\end{tabular}

M Mean, SD Standard Deviation, AN Anorexia nervosa, HC Healthy control, MDD Major depressive disorder, PTSD Posttraumatic stress disorder, OCD Obsessivecompulsive disorder, BDD Body dysmorphic disorder, EDE Eating Disorder Examination, EDI-2 Eating Disorder Inventory-2, BDI-II Beck Depression Inventory-II, STAI State-Trait Anxiety Inventory. ${ }^{a} n=28 .{ }^{b} n=32 .{ }^{c} n=34 .{ }^{d} n=26$. ${ }^{e}$ Multiple entries per person possible.

${ }^{*} p<.05 ;{ }^{* * *} p<.001$

Table 2 Associations of eating disorder-specific and general psychopathology with implicit drive for thinness in AN

\begin{tabular}{|c|c|c|c|c|c|c|c|c|c|c|}
\hline $\begin{array}{l}\text { D- } \\
\text { score }\end{array}$ & BMI & $\begin{array}{l}\text { EDE } \\
\text { Restraint }\end{array}$ & $\begin{array}{l}\text { EDE Eating } \\
\text { concern }\end{array}$ & $\begin{array}{l}\text { EDE Weight } \\
\text { concern }\end{array}$ & $\begin{array}{l}\text { EDE Shape } \\
\text { concern }\end{array}$ & $\begin{array}{l}\text { EDI-2 Drive } \\
\text { for thinness }\end{array}$ & $\begin{array}{l}\text { EDI-2 } \\
\text { Bulimia }\end{array}$ & $\begin{array}{l}\text { EDI-2 Body } \\
\text { dissatisfaction }\end{array}$ & $\begin{array}{l}\text { BDI-II } \\
\text { Total } \\
\text { score }\end{array}$ & $\begin{array}{l}\text { STAI Trait } \\
\text { anxiety }\end{array}$ \\
\hline pIAT & $\begin{array}{l}.178 \\
(p=.305)\end{array}$ & $\begin{array}{l}.299 \\
(p=.081)\end{array}$ & $\begin{array}{l}.407^{*} \\
(p=.015)\end{array}$ & $\begin{array}{l}.231 \\
(p=.181)\end{array}$ & $\begin{array}{l}.370^{*} \\
(p=.031)\end{array}$ & $\begin{array}{l}.244 \\
(p=.158)\end{array}$ & $\begin{array}{l}-.035 \\
(p=.844)\end{array}$ & $\begin{array}{l}.241 \\
(p=.163)\end{array}$ & $\begin{array}{l}.146 \\
(p=.401)\end{array}$ & $\begin{array}{l}.096 \\
(p=.588)\end{array}$ \\
\hline qIAT & $\begin{array}{l}-.136 \\
(p=.436)\end{array}$ & $\begin{array}{l}.379 * \\
(p=.025)\end{array}$ & $\begin{array}{l}.371^{*} \\
(p=.028)\end{array}$ & $\begin{array}{l}.371^{*} \\
(p=.028)\end{array}$ & $\begin{array}{l}.359 * \\
(p=.037)\end{array}$ & $\begin{array}{l}.437^{* *} \\
(p=.009)\end{array}$ & $\begin{array}{l}-.129 \\
(p=.459)\end{array}$ & $\begin{array}{l}.543^{* *} \\
(p=.001)\end{array}$ & $\begin{array}{l}.096 \\
(p=.583)\end{array}$ & $\begin{array}{l}.080 \\
(p=.654)\end{array}$ \\
\hline
\end{tabular}

AN Anorexia nervosa, pIAT Picture-based implicit association test, qIAT Questionnaire-based implicit association test, BMI Body mass index, EDE Eating Disorder Examination, EDI-2 Eating Disorder Inventory-2, BDI-II Beck Depression Inventory-2, STAI State-Trait Anxiety Inventory. ${ }^{*} p<.05 ;{ }^{* *} p<.01$ 
HCs $(M=-.61, S D=.40), t(59.38)=-3.87 ; p<.001 ; d=$ .94 (Fig. 1). Specifically, HCs showed a stronger association between underweight models and negative words compared to participants with AN. However, contrary to our first hypothesis, participants with AN did not exhibit positive biases toward underweight models; rather, the association was close to zero.

\section{Questionnaire-based implicit association test}

A significant group difference in the qIAT D-score between participants with $\mathrm{AN}(M=.28, S D=.54)$ and $\mathrm{HCs}$ $(M=-.29, S D=.33)$ emerged, $t(57.19)=-5.15 ; p<.001$; $d=1.24$ (Fig. 2). Specifically, the AN group exhibited a strong association between pro-dieting statements and true statements, whereas HCs exhibited a strong association between pro-dieting statements and false statements.

\section{Discussion}

This study examined associations of implicit measures of drive for thinness with explicit general and ED-specific psychopathological measures such as explicit drive for thinness in a German sample of participants with AN. Additionally, we examined differences in implicit attitudes toward thinness and dieting measured by the qIAT and pIAT between participants with AN and HCs, therewith partially replicating the US-American study by
Izquierdo et al. [33] as a proof of concept in a Germanlanguage sample.

Partly confirming our first hypothesis, the results of the correlation analyses within the AN group revealed that higher qIAT D-scores were associated with more pronounced ED-specific psychopathology. PIAT Dscores showed no significant associations with most EDspecific psychopathological measures except the EDE eating concern and EDE shape concern scores. The results of both IATs showed significantly greater implicit drive for thinness among participants with AN compared to HCs, confirming our second hypothesis and indicating that IATs can reliably assess implicit drive for thinness. Specifically, in the qIAT, the AN group revealed a stronger positive bias toward pro-dieting statements, while pIAT results indicated neither positive nor negative biases toward underweight models in the AN group, with their actual D-score value being close to zero.

We found significant correlations of qIAT D-scores with explicit drive for thinness and body dissatisfaction as well as with the EDE subscale scores across all participants with AN. While viewing the qIAT D-scores as a proxy measurement of implicit FP, these results would be consistent with previous research indicating a less severe ED-specific psychopathology in individuals with NFP-AN vs. FP-AN [10, 11, 14, 15]. This also supports

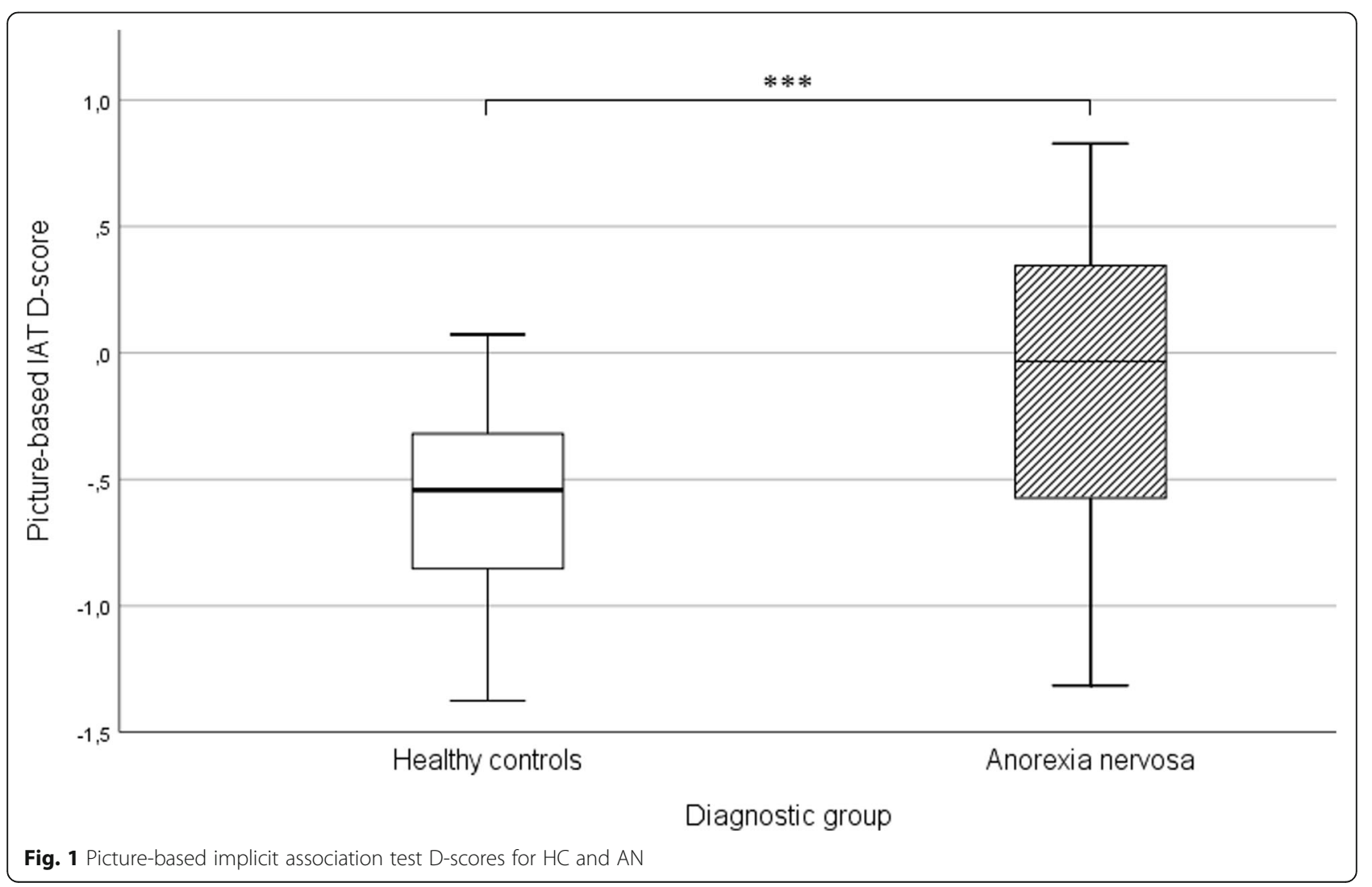




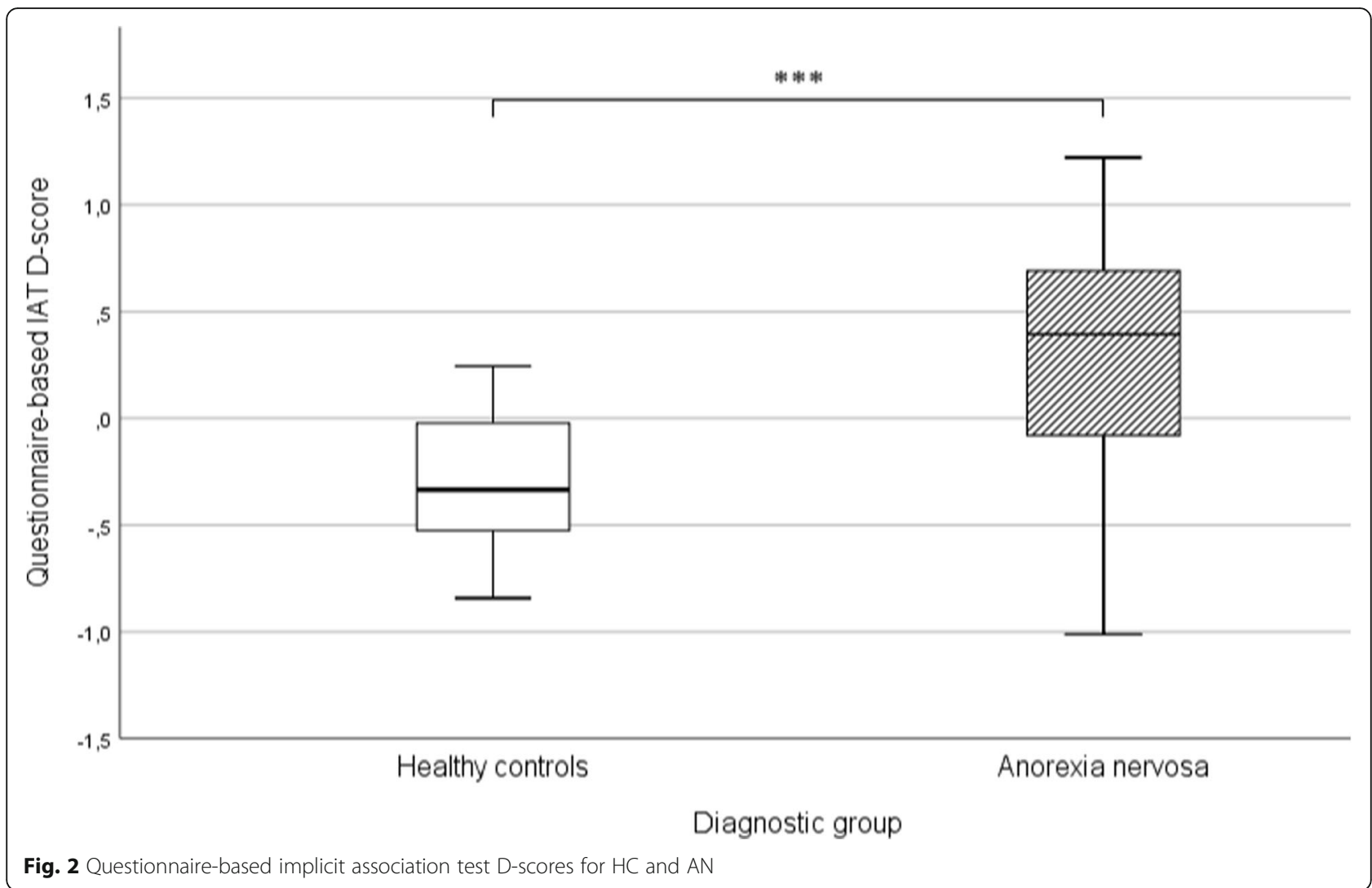

the approach that implicit FP represents a dimensional variable comparable to explicitly measured ED-specific psychopathology [36, 37]. The correlation of qIAT Dscores with the EDI-2 subscales drive for thinness and body dissatisfaction may additionally be explained by the proximity of the qIAT to those explicit measures due to relatedness of item content [33, 51], which might also be partly the case for EDE subscales restraint, eating, weight and shape concern [12]. The lack of correlations between the pIAT and most explicit ED-specific psychopathological measures could be explained by different types of indicators for the measured target construct [51]. Semantically complex stimuli, such as those in the qIAT, activate a wider range of self-referential psychological constructs, which then more closely correspond to the assessment in self-reports [51]. On a related note, while statements in the qIAT made it possible to map the thematic range of the four EDE subscales, the pIAT primarily addressed the shape concern subscale. Based on pictures, a viewer can only directly infer the figure of the person shown (not his or her weight), which may have boosted comparison processes in participants with greater degrees of FP. The correlative result regarding the eating concern subscale, however, is counterintuitive. Overall, however, these results are in line with the categorical findings by Izquierdo et al. [33].
Consistent with previous research [52], HCs showed positive associations between normal-weight models and positive words when confronted with underweight models in the pIAT, which could be interpreted as a healthy concept of body image ideal. In line with this, Izquierdo et al. [33] found a strong negative association between underweight models and positive words in HCs. In contrast, research regarding implicit biases in the AN group is heterogenous. While Ahern et al. [32] found that women with a stronger drive for thinness had positive implicit biases toward underweight models, Izquierdo et al. [33] observed a negative association toward underweight models in the AN group. Results of the present study showed a pIAT D-score value close to zero in the AN group, which can be interpreted as a lack of association [44]. This may be explained by an identification bias, insofar as women with AN display greater self-deprecating double standards in body fat rating than women without an ED [53]. Consequently, their perception may be distorted only when looking at their own bodies and not when looking at other bodies like in the pIAT. Izquierdo et al. [33] suggested that participants' socially established ideals of thinness do not correspond with the images of underweight models used in the pIAT, which might have led to less relevance for one's own body image and associated implicit biases. This is 
further supported by findings on implicit associations toward emaciation, suggesting that individuals with AN might display complex implicit biases toward thinness $[33,54]$. In order to investigate this approach, future pIATs should consider inlcuding pictures of participants' own vs. others' bodies-possibly in varying levels of thinness.

Average qIAT D-scores in $\mathrm{HCs}$ also match previous research [33] indicating an association between pro-dieting and false statements. This is in line with the abovementioned healthy approach toward underweight and normal-weight models in HCs. Consistent with previous research [31], the AN group presented a strong positive implicit bias toward pro-dieting statements in the qIAT. Compared to the pIAT results, the qIAT with its selfreferential statements might have led to a greater relevance for their own body images and implicit beliefs. In addition, Friedman et al. [51] support that the qIAT is a more appropriate measure of attitudes about the selfwith better convergent validity with explicit measurescompared to other implicit measures. Due to the increased self-relevance and proximity to actual attitudes, the qIAT seems to provide more information regarding the implicit measurement of drive for thinness [33].

In light of the categorical findings by Izquierdo et al. [33] that failed to reveal differences in implicit drive for thinness between individuals with AN reporting and not reporting FP, a significant relationship between implicit and explicit drive for thinness and other ED-specific measures seems contradictory. These inconsistencies may be attributable to changes in reasons for food intake restriction during the course of AN [55], besides fear of weight gain, e.g., need for control [56, 57]. As a consequence, we might have diverse samples of individuals with varying degrees of FP that can be better represented by dimensional metrics. Additionally, the consideration of FP and drive for thinness as one end of a single dimension, using the latter as sort of a proxy for the former, might also have contributed to the discussed inconsistencies. A recent study by Rodgers, DuBois, Frumkin, and Robinaugh [58] has shown that the frequently interchangeably measured concepts FP and drive for thinness seem to be distinct constructs and should not be considered as a single symptom. Rodgers and colleagues [58] further highlight that these constructs also have distinct association patterns within the network of ED symptoms. This assumption would lead to a high relevance of assessing those constructs separately in future studies, and, therewith, perhaps allow for categorical data to match our dimensional data.

Some limitations of this study must be acknowledged. Since the analyses of the current study have been performed in small samples, the generalizability of results to the AN population is limited. Moreover, 14 participants with AN had at least one comorbid mental disorder, and the impact of comorbidity on the results cannot be quantified. However, given that comorbidity is the rule rather than the exception in $\mathrm{AN}$, the recruitment procedure provided a representative group of the AN population. Furthermore, the IAT is susceptible to contextual influences known from explicit self-reports [23], although these are considerably reduced due to the improved evaluation algorithm on IATs and corresponding recommendations regarding the analysis [44]. The strengths of this study are the thorough diagnosis of all participants and the standardized application of IATs, allowing for a partial replication and substantial extension of the work by Izquierdo et al. [33].

\section{Conclusion}

The present study is one of few investigations of FP in AN with a dimensional approach based on an implicit task. Moreover, the present study partially replicated a study by Izquierdo et al. [33] which compared implicit associations between individuals with low-weight EDs. In regard to this categorical approach, the present findings are consistent with previous research. Thus, this study represents a proof-of-concept study for the implicit measurement of a construct that might serve to further tailor treatment strategies to individual needs based on the level of FP or drive for thinness. Future studies should employ the paradigm in the German version to assess samples of individuals with different levels of FP as has been done in an American sample [33]. Since in our sample only seven individuals could have been assigned to the subgroup NFP-AN, we refrained from this analysis.

Furthermore, our study underscores the importance of using a dimensional perspective on relevant ED-specific constructs, such as drive for thinness and FP in AN. Even if these constructs cannot be considered as congruent, they are closely related via superordinate concepts such as body dissatisfaction or negative body image. As part of this, the body functions as a self-value determining construct leading to food intake restriction. Therefore, intensive research on drive for thinness and FP as well as on its dimensional analyses remains of great importance. Future studies could focus on longitudinal analyses in AN using implicit measures to effectively monitor implicit manifestations and courses. If there are samples of individuals with lower degrees of or nonexistent FP, individually adapted treatment approaches such as the exploration and cognitive modification of alternative rationales for food intake restriction might be more promising. As a therapist, addressing unreported FP and taking these statements seriously could improve the therapeutic relationship as well as the patients' compliance, which in turn could also have a positive impact 
on increased drop-out rates and low insights into their condition that was previously found in individuals with NFP-AN [11]. For individuals lacking insight into their underlying FP, a treatment focused on the detection and regulation of emotions could increase treatment efficacy. Considering that a discrepancy between explicit and implicit attitudes can lead to a detrimental effect on change and intention to change, interventions should aim for a concordance of explicit and implicit attitudes. In individuals who lack conscious awareness of or deny underlying FP, implicit measurements could shed light on it and consequently support treatments to improve perception and communication of FP.

\section{Abbreviations \\ AN: Anorexia nervosa; ANOVA: Analysis of variance; ARFID: Avoidant/ restrictive food intake disorder; BDI-II: Beck Depression Inventory-II; BMI: Body mass index; DSM-IV/ -5: Diagnostic and Statistical Manual of Mental Disorders fourth edition/ fifth edition; ED: Eating disorder; EDE: Eating Disorder Examination; EDI-2: Eating Disorder Inventory second edition; FP: Fat phobia; Fear of weight gain; HC: Healthy Control; IAT: Implicit association test; NFP: Non-fat phobia; No fear of weight gain; pIAT: picture-based implicit association test; qIAT: questionnaire-based implicit association test; SCID: Structured Clinical Interview for DSM-IV; STAI(-T): State-Trait Anxiety Inventory (trait scale)}

\section{Acknowledgments}

N/a.

\section{Authors' contributions}

JJT, FP, and ASH designed the study, FP and ASH developed the paradigm. TB and NK collected data and were supervised by ASH. TB, NK, and ASH analysed the data, and all authors interpreted data. TB, NK, and ASH drafted the first version of the article, all authors critically revised the article and approved the final version.

\section{Funding}

The study was funded by a start-up grant from the Innovation and Research Pool of Osnabrück University to the last author

\section{Availability of data and materials}

The local ethics committee stipulated that data must not be passed on to third parties. Therefore, data sharing is not applicable to this article.

\section{Ethics approval and consent to participate}

All procedures performed in studies involving human participants were in accordance with the ethical standards of the Osnabrück University ethics committee and with the 1964 Helsinki declaration and its later amendments or comparable ethical standards.

Informed consent was obtained from all individual participants included in the study, and their legal guardians if minor.

\section{Consent for publication}

N/a.

\section{Competing interests}

The authors declare that they have no competing interests.

\section{Author details}

${ }^{1}$ Department of Psychiatry and Psychotherapy, University of Münster, Münster, Germany. ${ }^{2}$ Unit of Clinical Psychology and Psychotherapy, Institute of Psychology, Osnabrück University, Knollstr. 15, 49069 Osnabrück, Germany. ${ }^{3}$ Eating Disorders Clinical and Research Program, Department of Psychiatry, Massachusetts General Hospital and Harvard Medical School, Boston, MA, USA. ${ }^{4}$ Neuroendocrine Unit, Department of Medicine, Massachusetts General Hospital and Harvard Medical School, Boston, MA, USA.
Received: 8 July 2020 Accepted: 9 February 2021

Published online: 18 February 2021

\section{References}

1. Arcelus J, Mitchell AJ, Wales J, Nielsen S. Mortality rates in patients with anorexia nervosa and other eating disorders. A meta-analysis of 36 studies. Arch Gen Psychiatry. 2011;68:724-31. https://doi.org/10.1001/a rchgenpsychiatry.2011.74.

2. Murray SB, Quintana DS, Loeb KL, Griffiths S, Le Grange D. Treatment outcomes for anorexia nervosa: a systematic review and meta-analysis of randomized controlled trials. Psychol Med. 2019;49:701-4. https://doi.org/1 $0.1017 /$ S0033291718002088.

3. Zeeck A, Herpertz-Dahlmann B, Friederich HC, Brockmeyer T, Resmark G, Hagenah $U$, et al. Psychotherapeutic treatment for anorexia nervosa: a systematic review and network meta-analysis. Front Psychiatry. 2018:9:158. https://doi.org/10.3389/fpsyt.2018.00158.

4. Legenbauer T, Vocks S. Manual der kognitiven Verhaltenstherapie bei Anorexie und Bulimie. Berlin: Springer; 2014. https://doi.org/10.1007/978-3642-20385-5.

5. Forrest LN, Jones PJ, Ortiz SN. Core psychopathology in anorexia nervosa and bulimia nervosa: a network analysis. Int J Eat Disord. 2018;51:668-79. https://doi.org/10.1002/eat.22871.

6. Heider N, Spruyt A, De Houwer J. Body dissatisfaction revisited: on the importance of implicit beliefs about actual and ideal body image. Psychologica Belgica. 2018;57:158-73. https://doi.org/10.5334/pb.362.

7. Williamson DA, White MA, York-Crowe E, Stewart TM. Cognitive-behavioral theories of eating disorders. Behav Modif. 2004;28:711-38. https://doi.org/1 $0.1177 / 0145445503259853$.

8. American Psychiatric Association. Diagnostic and statistical manual of mental disorders. 4th ed. Washington DC: American Psychiatric Association; 1994

9. American Psychiatric Association. Diagnostic and statistical manual of mental disorders. 5th ed. Washington DC: American Psychiatric Association; 2013. https://doi.org/10.1176/appi.books.9780890425596.

10. Ramacciotti CE, Dell'Osso L, Paoli RA, Ciapparelli A, Coli E, Kaplan AS, Garfinkel PE. Characteristics of eating disorder patients without a drive for thinness. Int J Eat Disord. 2002;32:206-12. https://doi.org/10.1002/eat.10067.

11. Santonastaso P, Bosello R, Schiavone P, Tenconi E, Degortes D, Favaro A. Typical and atypical restrictive anorexia nervosa: weight history, body image, psychiatric symptoms, and response to outpatient treatment. Int J Eat Disord. 2009;42:464-70. https://doi.org/10.1002/eat.20706.

12. Hilbert A, Tuschen-Caffier B. Eating Disorder Examination: Deutschsprachige Übersetzung. 2nd ed. Tübingen: dgvt-Verlag; 2016.

13. Paul T, Thiel A. Eating disorder Inventory-2: EDI-2. Göttingen: Hogrefe; 2005.

14. Dalle Grave R, Calugi S, Marchesini G. Underweight eating disorder without over-evaluation of shape and weight: atypical anorexia nervosa? Int J Eat Disord. 2008;41:705-12. https://doi.org/10.1002/eat.20555.

15. Carter JC, Bewell-Weiss CV. Nonfat phobic anorexia nervosa: clinical characteristics and response to inpatient treatment. Int J Eat Disord. 2011;44 220-4. https://doi.org/10.1002/eat.20820.

16. Murray HB, Coniglio K, Hartmann AS, Becker AE, Eddy KT, Thomas JJ. Are eating disorders "all about control?" the elusive psychopathology of nonfat phobic presentations. Int J Eat Disord. 2017;50:1306-12. https://doi.org/10.1 002/eat.22779.

17. Crow SJ, Swanson SA, Peterson CB, Crosby RD, Wonderlich SA, Mitchell JE. Latent class analysis of eating disorders: relationship to mortality. J Abnorm Psychol. 2012;121:225-31. https://doi.org/10.1037/a0024455.

18. Thomas JJ, Hartmann AS, Killgore WDS. Non-fat-phobic eating disorders: why we need to investigate implicit associations and neural correlates. Int J Eat Disord. 2013;46:416-9. https://doi.org/10.1002/eat.22098.

19. Lulé D, Schulze UE, Bauer K, Schöll F, Müller S, Fladung A, Uttner I. Anorexia nervosa and its relation to depression, anxiety, alexithymia and emotional processing deficits. Eat Weight Disord. 2014;19:209-16. https://doi.org/10.1 007/s40519-014-0101-z.

20. Konstantakopoulos G, Tchanturia K, Surguladze SA, David AS. Insight in eating disorders: clinical and cognitive correlates. Psychol Med. 2011;41: 1951-61. https://doi.org/10.1017/S0033291710002539.

21. Garner DM. EDI-2. Eating disorder Inventory-2. Professional manual. Odessa: Psychological Assessment Resources; 1991.

22. Cooper $Z$, Fairburn $C$. The eating disorder examination: a semi-structured interview for the assessment of the specific psychopathology of eating 
disorders. Int J Eat Disord. 1987;6:1-8. https://doi.org/10.1002/1098-108X(1 98701)6:1<1::AID-EAT2260060102>3.0.CO;2-9.

23. De Houwer J. The implicit association test as a tool for studying dysfunctional associations in psychopathology: strengths and limitations. Behav Ther Exp Psychiatry. 2002;33:115-33. https://doi.org/10.1016/S00057916(02)00024-1.

24. Greenwald AG, McGhee DE, Schwartz JKL. Measuring individual differences in implicit cognition: the implicit association test. J Pers Soc Psychol. 1998; 74:1464-80. https://doi.org/10.1037/0022-3514.74.6.1464.

25. Blair IV. The malleability of automatic stereotypes and prejudice. Personal Soc Psychol Rev. 2002;6:242-61. https://doi.org/10.1207/S15327957PSPR0603_8.

26. De Jong PJ, Pasman W, Kindt M, Van den Hout MA. A reaction time paradigm to assess (implicit) complaint-specific dysfunctional beliefs. Behav Res Ther. 2001;39:101-13. https://doi.org/10.1016/50005-7967(99)00180-1.

27. Teachman BA, Gregg AP, Woody SR. Implicit associations for fear-relevant stimuli among individuals with snake and spider fears. J Abnorm Psychol. 2001;110:226-35. https://doi.org/10.1037/0021-843X.110.2.226.

28. Clerkin EM, Teachman BA, Smith AR, Buhlmann U. Specificity of implicitshame associations: comparison across body dysmorphic, obsessivecompulsive, and social anxiety disorders. Clin Psychol Sci. 2014;2:560-75. https://doi.org/10.1177/2167702614524944.

29. Ricceri A, Fronza S, Galimberti E, Bellodi L. Implicit associations in eating disorders: an experimental study using implicit associations test. Eur Psychiatry. 2011;26:721. https://doi.org/10.1016/S0924-9338(11)72426-9.

30. Spring VL, Bulik CM. Implicit and explicit affect toward food and weight stimuli in anorexia nervosa. Eat Behav. 2014;15:91-4. https://doi.org/10.1016/ j.eatbeh.2013.10.017.

31. Vartanian LR, Herman CP, Polivy J. Implicit and explicit attitudes toward fatness and thinness: the role of the internalization of societal standards. Body Image. 2005;2:373-81. https://doi.org/10.1016/j.bodyim.2005.08.002.

32. Ahern AL, Bennett KM, Hetherington MM. Internalization of the ultra-thin ideal: positive implicit associations with underweight fashion models are associated with drive for thinness in young women. Eat Disord. 2008;16: 294-307. https://doi.org/10.1080/10640260802115852.

33. Izquierdo A, Plessow F, Becker KR, Mancuso CJ, Slattery M, Murray HB, et al. Implicit attitudes toward dieting and thinness distinguish fat-phobic and non-fat-phobic anorexia nervosa from avoidant/restrictive food intake disorder in adolescents. Int J Eat Disord. 2019;52:419-27. https://doi.org/10.1 002/eat.22981.

34. Yovel I, Friedman A. Bridging the gap between explicit and implicit measurement of personality: the questionnaire-based implicit association test Pers Individ Differ. 2013;54:76-80. https://doi.org/10.1016/j.paid.2012.08.015.

35. Becker AE, Thomas JJ, Pike KM. Should non-fat-phobic anorexia nervosa be included in DSM-V? Int J Eat Disord. 2009;42:620-35. https://doi.org/10.1002/ eat.20727.

36. Williamson DA, Gleaves DH, Stewart TM. Categorical versus dimensional models of eating disorders: an examination of the evidence. Int J Eat Disord. 2005;37:1-10. https://doi.org/10.1002/eat.20074.

37. Jennings KM, Bodell LP, Crosby RD, Haynos AF, Wildes JE. Mixture modeling to characterize anorexia nervosa: integrating personality and eating disorder psychopathology. J Am Psychiatr Nurses Assoc. 2019. https://doi.org/10.11 77/1078390319862029.

38. Hartmann AS, Thomas JJ, Wilson AC, Wilhelm S. Insight impairment in body image disorders: delusionality and overvalued ideas in anorexia nervosa versus body dysmorphic disorder. Psychiatry Res. 2013;210:1129-35. https:// doi.org/10.1016/.jpsychres.2013.08.010

39. Morgan JF, Reid F, Lacey JH. The SCOFF questionnaire: assessment of a new screening tool for eating disorders. Br Med J. 1999;319:1467-8. https://doi. org/10.1136/bmj.319.7223.1467.

40. Wittchen HU, Zaudig M, Fydrich T. SKID - Strukturiertes klinisches Interview für DSM-IV: Achse I und Achse II. Göttingen: Hogrefe; 1997.

41. Beesdo-Baum K, Zaudig M, Wittchen HU. SCID-5-CV. Strukturiertes Klinisches Interview für DSM-5๑ -Störungen - Klinische Version. Göttingen: Hogrefe; 2019.

42. Korn J, Vocks S, Rollins LH, Thomas JJ, Hartmann AS. Fat-phobic and non-fat-phobic anorexia nervosa: a conjoint analysis on the importance of shape and weight. Front Psychol. 2020;11:90. https://doi.org/10.3389/ fpsyg.2020.00090.

43. Hartmann AS, Borgers T, Thomas JJ, Giabbiconi CM, Vocks S. Faced with one's fear: Attentional bias in anorexia nervosa and healthy individuals upon confrontation with an obese body stimulus in an eye-tracking paradigm. Brain Behavior. 2020. https://doi.org/10.1002/brb3.1834.
44. Greenwald AG, Nosek BA, Mahzarin RB. Understanding and using the implicit association test: I. an improved scoring algorithm. J Pers Soc Psychol. 2003;85:197-216. https://doi.org/10.1037/0022-3514.85.2.197.

45. Nosek BA, Greenwald AG, Banaji MR. Understanding and using the implicit association test: II. Method variables and construct validity. Personal Soc Psychol Bull. 2005;31:166-80. https://doi.org/10.1177/0146167204271418.

46. Berger U, Wick K, Hölling H, Schlack R, Bormann B, Brix C, Sowa M, Schwartze $D$, Strauß B. Screening of disordered eating in 12-year-old girls and boys: psychometric analysis of the German versions of SCOFF and EAT-26. Psychother Psych Med. 2011;61:311-8. https://doi.org/10.1055/s-0031-1271786.

47. Richter F, Strauß B, Brähler E, Adametz L, Berger U. Screening disordered eating in a representative sample of the German population: usefulness and psychometric properties of the German SCOFF questionnaire. Eat Beh. 2017; 25:81-8. https://doi.org/10.1016/j.eatbeh.2016.06.022.

48. Hautzinger M, Keller F, Kühner C. Beck depressions-Inventar (BDI-II): revision. Frankfurt: Harcourt Test Services; 2006.

49. Laux L, Glanzmann P, Schaffner P, Spielberger CD. Das state-trait Angstinventar. Göttingen: Beltz Test; 1981.

50. Cohen J. The effect size index: d. statistical power analysis for the behavioral sciences. 2nd ed. Hillsdale: Lawrence Erlbaum Associates; 1988.

51. Friedman A, Katz BA, Cohen IH, Yovel I. Expanding the scope of implicit personality assessment: an examination of the questionnaire-based implicit association test (q|AT). J Pers Assess. 2020;20:1-12. https://doi.org/10.1080/ 00223891.2020 .1754230

52. Marini M. Underweight vs. overweight/obese: Which weight category do we prefer? Dissociation of weight-related preferences at the explicit and implicit level. Obes Sci Pract. 2017;3:390-8. https://doi.org/10.1002/osp4.136.

53. Voges MM, Giabbiconi CM, Schöne B, Braks K, Huber TJ, Waldorf M, Hartmann AS, Vocks S. Double standards in body evaluation? How identifying with a body stimulus influences ratings in women with anorexia nervosa and bulimia nervosa. Int J Eat Disord. 2018;51:1223-32. https://doi. org/10.1002/eat.22967.

54. Smith AR, Joiner TE Jr, Dodd DR. Examining implicit attitudes toward emaciation and thinness in anorexia nervosa. Int J Eat Disord. 2014;47:13847. https://doi.org/10.1002/eat.22210.

55. Ngai ESW, Lee S, Lee AM. The variability of phenomenology in anorexia nervosa. Acta Psychiatr Scand. 2000;102:314-7. https://doi.org/10.1034/j.1 600-0447.2000.102004314.x

56. Fairburn CG, Cooper Z, Shafran R. Cognitive behaviour therapy for eating disorders: a 'transdiagnostic' theory and treatment. Behav Res Ther. 2003;41: 509-28. https://doi.org/10.1016/S0005-7967(02)00088-8.

57. Vervaet $M$, Van Heeringen $C$, Audenaert $K$. Is drive for thinness in anorectic patients associated with personality characteristics? Eur Eat Disord Rev. 2004:12:375-9. https://doi.org/10.1002/erv.586.

58. Rodgers RF, DuBois R, Frumkin MR, Robinaugh DJ. A network approach to eating disorder symptomatology: do desire for thinness and fear of gaining weight play unique roles in the network? Body Image. 2018;27:1-9. https:// doi.org/10.1016/.bodyim.2018.07.004

\section{Publisher's Note}

Springer Nature remains neutral with regard to jurisdictional claims in published maps and institutional affiliations.

Ready to submit your research? Choose BMC and benefit from

- fast, convenient online submission

- thorough peer review by experienced researchers in your field

- rapid publication on acceptance

- support for research data, including large and complex data types

- gold Open Access which fosters wider collaboration and increased citations

- maximum visibility for your research: over $100 \mathrm{M}$ website views per year

At $\mathrm{BMC}$, research is always in progress.

Learn more biomedcentral.com/submissions 